\title{
Molecular Dynamics Simulations of Bottle-Brush Polymers with a Flexible Backbone under Theta and Good Solvent Conditions
}

\author{
Panagiotis E. Theodorakis ${ }^{1,2, *}$, Nikolaos G. Fytas ${ }^{3}$ \\ ${ }^{1}$ Faculty of Physics,University of Vienna A-1090, Boltzmann gasse 5, Vienna, Austria \\ ${ }^{2}$ Institute for Theoretical Physics and Center for Computational MaterialsScience (CMS), Technical University of Vienna A-1040 Vienna, \\ Hauptstrasse8-10, Austria \\ ${ }^{3}$ Departamento de Fisica Teorica I, Universidad Complutense de Madrid, Madrid, 28040, Spain
}

\begin{abstract}
Using molecular dynamics simulations of a standard bead-spring model for polymer chains,bottle-brush polymers with a flexib le backbone of $\mathrm{N}_{\mathrm{b}}$ effective units, where side chains of length $\mathrm{N}$ are grafted under theta and good solvent conditions in the range $T=3.0-4.0 \varepsilon / k_{B}$, are studied.The range of backbone and side chains' length varies correspondingly as $50 \leq N_{b} \leq 200$ and $5 \leq N \leq 40$ for two different grafting densities $\sigma$, namely $\sigma=0.5$ and 1.0 . Even at temperatures $\mathrm{T}$ close to the theta point the side chains are significantly stretched, as it has been confirmed for bottle brushes with a rigid backbone, their linear dimension depending on the solvent quality only weakly. However, the distribution of monomers shows a more pronounced dependence, which we characterize through the asphericity and acylindricity as functions of $\sigma$, T, $\mathrm{N}_{\mathrm{b}}$, and $\mathrm{N}$. In particular, increase of $\sigma, \mathrm{T}, \mathrm{N}_{\mathrm{b}}$, and $\mathrm{N}$ increases the normalized asphericity and acylindricity of the macromolecule. Interestingly, we also find that the dimensions of the side chains reveals differences in the distributions of side chain monomers by changing the backbone length $\mathrm{N}_{\mathrm{b}}$ as the region between the two backbone-ends increases. A method to extract the persistence length of bottle-brush macromolecules and its drawbacks is also discussed given that different measures of the persistence length are not mutually consistent with each other and depend distinctly both on $\mathrm{N}_{\mathrm{b}}$ and the solvent quality.
\end{abstract}

Keywords Molecular Dynamics, Bottle-Brush Polymers, Structural Properties

\section{Introduction}

Macromolecules which consist of a backbone where side chains are graftedrandomly or regularly have recently found much interest[1-6]. Such macromolecules are described in terms of their structure by a multitudeof parameters, such as the backbone length $\mathrm{N}_{\mathrm{b}}$ and the grafting densitythat the side chains with length $\mathrm{N}$ are grafted ontothe flexible backbone, while solvent conditions may also varyby variation of the temperature $\mathrm{T}$ or the $\mathrm{pH}$ of the solution resulting inthe structural change of these stimuli-responsive macromolecules. The response of the large scale structure of bottle-brush polymers tosolvent conditions is an intriguing

Biopolymers with a related architecture are also abundant in nature; for example proteoglycans [8] or the aggrecan molecules kept responsible for the very good lubricating properties in human joints[9]. In thiscontext the change in the solvent conditions is an important parameter, and the

* Corresponding author:

panagiotis.theodorakis@univie.ac.at (Panagiotis E. Theodorakis)

Published online at http://journal.sapub.org/ajcmp

Copyright (C) 2012 Scientific \& Academic Publishing. All Rights Reserved influence of these parameters on bottle-brush polymers has beenstudiedfor the case of single[10-12] and two-component[12-14] bottle brushes with a rigid backbone,suggesting in agreement with theoretical predictions structures rangingfrom individual collapsed chains at low grafting densities to the so-called "pearl-necklace" structures for intermed iate densities andto homogenous cylinders and Janus-like structures at even higher densities.

Another interesting discussion regards the local "stiffness" traditionally measured by the persistence length $1_{p}$ and the effective contour length[15-18].It is argued that finding a unique persistencelength measuring the "intrinsic" stiffness of a polymer cannotbe defined in the standard fashion with definitions that would all agree for Gaussian chains. Therefore, it has recently been shown that the persistence length depends not onlyon the backbone length[19,20], but on the solvent conditions as well [15]. Although there exist many experimental and theoretical studies for the linear dimensions of these macromolecules in various solvents[3,4,19,21-36], there are very few systematic studies of this problem[15], wherethe power laws and the associated effective exponents have been 
discussed.It has been shown that for bottle brushes with a flexible backbone evenat the theta point the side chains are considerably stretched, their linear dimension depending on the solvent quality only weakly, whilethe dependence of the persistence length on backbone length and temperature has alsobeen discussed[15].

The present work intends to make a contribution in giving a geometrical intuitionof single-component bottle-brush polymers with a flexible backbone undertheta and good solvent conditions and how the distribution of monomers changesby changing the various parameters. A pertinent discussion for a method to compute the persistence lengthof these complex macromolecules and its drawbacks will be also presented.The rest of this paper is laid out as follows. In Section 2,the current simulation model and its analysis are unfolded. A relevantdiscussion for the peculiarities of our system of interest is also provided. Section 3 presents a brief discussion of properties and our numerical results. This manuscript closes in Section 4 , with a short summary.

\section{Model and Methods}

We describe the backbone chain and the side chains by a bead-spring model[37-43] where all beads interact with a truncated and shifted Lennard-Jones $(\mathrm{LJ})$ potential $\mathrm{U}_{\mathrm{LJ}}(\mathrm{r})$ and nearest neighbours bonded together along a chain also experience the finitely extensible nonlinear elastic potential $\mathrm{U}_{\mathrm{FENE}}(\mathrm{r}), \mathrm{r}$ being the distance between the beads. Thus,

$$
U_{L J}(r)=\left\{\begin{array}{c}
4 \varepsilon_{L J}\left[\left(\sigma_{L J} / r\right)^{12}-\left(\sigma_{L J} / r\right)^{6}\right] \\
0, r>r_{c}
\end{array}+C, r \leq r_{c},\right.
$$

wherer $_{\mathrm{c}}=2.5 \sigma_{\mathrm{LJ}}$. The constant $\mathrm{C}$ is defined such that $\mathrm{U}_{\mathrm{LJ}}(\mathrm{r}$ $=r_{c}$ ) is continuous at the cut-off. Henceforth, units are chosen such that $\varepsilon_{L J}=1, \sigma_{\mathrm{LJ}}=1$, the Boltzmann constant $\mathrm{k}_{\mathrm{B}}$ $=1$, and in addition also the mass $m_{L J}$ ofbeads is chosen to be unity. The potential of Eq. (1) acts between any pair of beads, irrespective of whether they are bonded or not. For bonded beads additionally the potential $\mathrm{U}_{\mathrm{FENE}}(\mathrm{r})$ is used,

$$
U_{\text {FENE }}(r)=-\frac{1}{2} k r_{0}^{2} \ln \left[1-\left(r / r_{0}\right)^{2}\right], 0<r \leq r_{0},
$$

where the standard choice of parameters[40] $\left(\mathrm{r}_{0}=1.5\right.$ and $\mathrm{k}=30$ ) was adopted, and $\mathrm{U}_{\mathrm{FENE}}\left(\mathrm{r}>\mathrm{r}_{0}\right)=\infty$. Note that in our model there is no difference in interactions, irrespective of whether the considered beads are effective monomers of the backbone or of the side chains, implyingthat the polymer forming the backbone is either chemicallyidentical to the polymers that are tethered as side chains tothe backbone, or at least on coarse-grained length scales asconsidered here the backbone and side chain poly mers are nolonger distinct. There is also no difference between the bondlinking the first monomer of a side chain to monomer ofthe backbone and bonds between any other pairs of bondedmonomers. Of course, our study does not address any effectsdue to a particular chemistry relating to the synthesis of thesebottle-brush polymers, but as usually done[40,44,45], we address universal features of the conformational properties of thes emacromo lecules.

There is one important distinction relating to our previouswork[10-14] on bottle-brush polymers with rigid backbones: followingGrest and Murat[40], there the backbone was taken as aninfinitely straight line in continuous space, thus allowingarbitrary values of the distances between neighbouring graftingsites, and hence the grafting density $\sigma$ could be continuouslyvaried. For the present model, where we disregard anypossible quenched disorder resulting from the grafting process, ofcourse, the grafting density $\sigma$ is quantized: we denote here by $\sigma=1$ the case that every backbone monomer carries a sidechain, $\sigma=0.5$ means that every second backbone monomer hasa side chain, etc. Chain lengths of side chains were chosenas $\mathrm{N}=5,10,20$, and 40 , while backbone chain lengths were chosen as $\mathrm{N}_{\mathrm{b}}=50,100$, and 200 , respectively.

It is obvious, of course, that for such short side chainlengths any interpretation of characteristic lengths in terms of power laws, such as $R_{c} \propto N^{v_{e f f}}$, is a delicate matter, $v_{\mathrm{eff}}$ being an effective exponent and characterizes only the specifiedrange of rather s mall values of $\mathrm{N}$ and not the limit $N \rightarrow \infty$ considered by most theories[6,25,33-35]. Thus, the actual value of $v_{\text {eff }}$ is generally of limited interest, it only gives an indication to whichpart of an extended crossover region the data belong. However,we emphasize that: (i) our range of $\mathrm{N}$ nicely corresponds tothe range available in experiments[1,2,21-23,32,46-48] and (ii) the analys is in term of power laws with effective exponents isa standard practice of experimentalists in this context.A simulation analysis for the static properties of bottle-brushmacromolecules with flexible backbone have been discussed in detail previously[15].In this work we rather focus on the overall shapes that suchmacro molecules obtain, and discuss various aspects that couldnot be discussed in the frame of effective exponent analysis.

We recall that for linear chains, the theta temperaturefor the present (implicit solvent) model has been roughlyestimated[46] as $\mathrm{T}_{\text {theta }} \approx 3.0$ (note, however, that there is still some uncertainty about the precise value of $\mathrm{T}_{\text {theta, }}$, for a similar model[47] $\mathrm{T}_{\text {theta }}=3.18$ in this case, couldonly be established for chain lengths exceeding $\mathrm{N}=$ 200).Thus, in the present work we have thoroughly studied thetemperature range $3.0 \leq T \leq 4.0$. From previous work[48] on rather long chains in polymer brushes on flat surfaces, using the same model[Eqs. (1) and (2)] to describe the interactions, it is known that for $\mathrm{T}=4.0$ one finds $\mathrm{a}$ behaviour characteristicfor (moderately) good solvents. Very good solvent conditionscould be obtained from a slightly different model that has extensively been studied for standard polymer brushes [40,49],where the cut-off in Eq. (1) is chosen to coincide with theminimum of the potential, $r_{c}=2^{1 / 6} \sigma_{L J}$ (and then also $\mathrm{T}=1$ can be chosen for this essentially a-thermal model).

In our simulations, the positions $\left\{r_{i}(t)\right\}$ of the effective monomers with label $\mathrm{i}$ evolve in time $\mathrm{t}$ according toNewton's equation of motion, amended by the 
Langevinthermostat[37-49]

$$
m \frac{d^{2} r_{i}}{d t^{2}}=-\nabla U_{i}-\gamma \frac{d r_{i}}{d t}+\Gamma_{i}(t),
$$

where $\mathrm{U}_{\mathrm{i}}$ is the total potential acting on the $\mathrm{i}$-th bead due to its interactions with the other beads at sites $\left\{r_{j}(t)\right\}, \gamma$ is the friction coefficient, and $\Gamma_{\mathrm{i}}(\mathrm{t})$ is the associated random force. The latter is related to $\gamma$ by the fluctuation-dis sipationrelation

$$
\left\langle\Gamma_{i}(t) \cdot \Gamma_{j}\left(t^{\prime}\right)\right\rangle=6 k_{B} T \delta_{i j} \delta\left(t-t^{\prime}\right) .
$$

Following previous work[37-49], we choose $\gamma=0.5$, the MDtime unit

$$
\tau_{i j}=\left(\frac{m_{i j} \sigma_{L J}^{2}}{\varepsilon_{i j}}\right)^{1 / 2},
$$

beingalso unity, for our choice of units. Equation (3) was integrated using the leap from algorith $\mathrm{m}[50]$, with a time step $\delta t=0.006 \tau_{L J}$, and utilizing the GROMACS package[51]. For the calculation of properties of the bottle brushes, typically 500 statistically independent configurations are averaged over. Of course, for bottle-brushes with large $\mathrm{N}_{b}$ equilibration of the polymer conformations is a difficult problem. Since we expect that end-to-end distance $R_{e}$ and gyration radius $R_{g}$ of the whole molecule belong to the slowest relaxing quantities, the judgment of the quality of results was based on the autocorrelations function of either of these quantities [15].

\section{Results and Discussion}

The interplay of various length scales in bottle-brush macromolecules results in interesting structures. The most extreme cases for the present system are shown in Fig. 1. For small values of $\mathrm{N}$ and temperatures close to theta $(\mathrm{T}=3.0)$, the molecule can adopt conformations like the one of Fig. 1a, or that of Fig. 1b where still locally the backbone holds a high local flexib ility. Moreover, at higher temperatures (e.g., $\mathrm{T}=4.0$, Figs. 1c and d) the whole macro molecule stretches due to the high affin ity with the solvent molecules (Figs. 1c and $\mathrm{d})$. Due to the longer side chains $(\mathrm{N}=40$, Fig. 1c) the backbone end beads are now not able to come close to each other due to the presence of the side chains which stretch the backbone monomers in the directions parallel to the backbone ends. It is clear that the side chains cause a significant stiffening of the backbone, at least on a coarse-grained scale, and that bottle brushes where $\mathrm{N}_{\mathrm{b}}$ is not very much larger than $\mathrm{N}$ look like wormlike chains. It has been shown that, for temperatures close to the theta temperature, bottle-brush molecules can be very well described by the Kratky-Porodmodel[15], which describes the crossover from rods to Gaussian chains. Such analys is was based on the discussion of bond orientationcorrelations along the backbone beads, the measurement of the end-to-end distance and the use of effective exponents[15].

Here, we show plots (Figs. 2 -5) of an alternative definition of a "local" persistence length $\mathrm{l}_{\mathrm{p}}(\mathrm{k})$ with the bond vector $\alpha_{k}$ connecting monomers at positions $r_{k}$ and $r_{k-1}\left(\alpha_{k}=\right.$ $\left.\mathrm{r}_{\mathrm{k}}-\mathrm{r}_{\mathrm{k}-1}\right)[16,30,52]$

$$
l_{p}(k)=l_{b}\left\langle\alpha_{k} \cdot R_{e} /\left|\alpha_{k}\right|^{2}\right\rangle
$$

but in the case of SAW chains [52]

$$
l_{p}(k) \approx c l_{b}\left[k\left(N_{b}-k\right) / N_{b}\right]^{2 v-1} .
$$

However, only the pre-factor c can be taken as a measure of intrinsic stiffness, but not $1_{p}(k)$ itself, since $l_{p}(k)$ exhibits maximum at $\mathrm{N}_{\mathrm{b}} / 2$ which diverges to infin ity proportional to $N_{b}^{2 v-1}$ as $N_{b} \rightarrow \infty[4,52]$. Furthermore, this problem is not improved when one considers an average of $l_{p}(k)$ along the chain $[5,16]$, while no divergence occurs for $1_{p}(1)[16]$. However, the use of $l_{p}(1)$ is inconvenient in simulations due to the limited statistical accuracy. However, it is interesting to see that this definition in agreement with previous work[15] gives distinct dependence of the "persistence length" both on $\mathrm{N}_{\mathrm{b}}$ and thesolvent quality. a
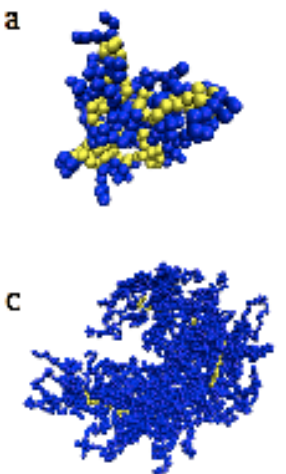

b

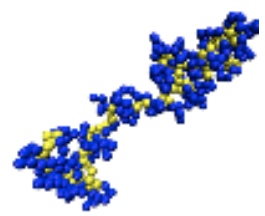

d

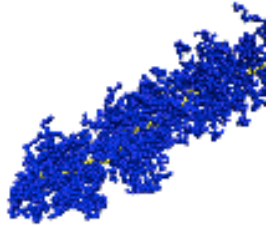

Figure 1. (Colour online) Selected snapshot pictures of equilibrated configurat ions of bottle-brush polymers. Backbonemonomers (when visible) are displayed in yellow (light grey) colour, side chain monomers in blue (darker grey). Cases a and b refer to $\sigma=0.5, \mathrm{~N}_{b}=100, \mathrm{~N}=5, \mathrm{~T}=3.0$. Cases $\mathrm{c}$ and $\mathrm{d}$ refer to $\sigma=1.0, \mathrm{~N}_{\mathrm{b}}=100, \mathrm{~N}=40, \mathrm{~T}=4.0$. Cases $\mathrm{a}, \mathrm{b}$, $\mathrm{c}$, and d give a good idea of the range of structures one obtains for bottle-brush polymers with flexible backbone under theta and good solvent conditions

In the case of $\sigma=0.5$ (Figs. 2 and 3) the data are rather exhibit some noise due to the lower "local" stiffness of the backbone. As discussed, one could define a "persistence length" from the maximum of the curvesthat corresponds exactly to the centre of the chain $\left(\mathrm{k} / \mathrm{N}_{\mathrm{b}}=0.5\right)$. It can be seen that for all $\mathrm{N}$ the increase of the temperature increases the stiffness ofthe backbone. Increase of oalso increases considerably thebackbonestiffness (Figs. 4 and 5). The persistence length depends on $\mathrm{N}, \mathrm{N}_{\mathrm{b}}, \sigma$, and $\mathrm{T}$.

The resulting estimates for a persistence length do not only depend on side chain length $\mathrm{N}$ and grafting density $\sigma$, but also on backbone chain length $\mathrm{N}_{\mathrm{b}}$ and on temperature $\mathrm{T}$ making difficult a consistent analysisfor this quantity. However, when onestudies the variation of the end-to-end distance of the backbone[15] for $\mathrm{T}=\mathrm{T}_{\Theta}$, an analys is in terms of the Kratky-Porodwormlike chain model beco mes feasible. But in this case one must identify the contour length $\mathrm{L}$ implied by this model with the "chemical" contour length $\mathrm{L}_{\mathrm{ch}}=\mathrm{N}_{\mathrm{b}} \mathrm{l}_{\mathrm{b}}$, where $\mathrm{l}_{\mathrm{b}}$ is the actual bond length, but ratherone has $L=N_{b} l_{b}{ }^{\text {eff }}$ with $l_{b}{ }^{\text {eff }}$ distinctly smaller than $l_{b}$. 
This effect results from the flexibility of the backbone on small scales; onlyon the scale of several backbone bonds does the stiffening due to the mutualside chains repulsions come into play. Thus, at the theta point both an effective contour length $\mathrm{L}$ and a persistence length $l_{\mathrm{p}}(\mathrm{k})$ are well-defined quantities, in terms of a fit of the data to the Kratky-Porod model, while under goodsolvent conditions such an analysis is not appropriate. In summary,an increase of $\sigma, \mathrm{N}$, and $\mathrm{T}$ results in an increase of the effective persistence length along the backbone. Effects of the $\mathrm{N}_{\mathrm{b}}$ willbe better described below.

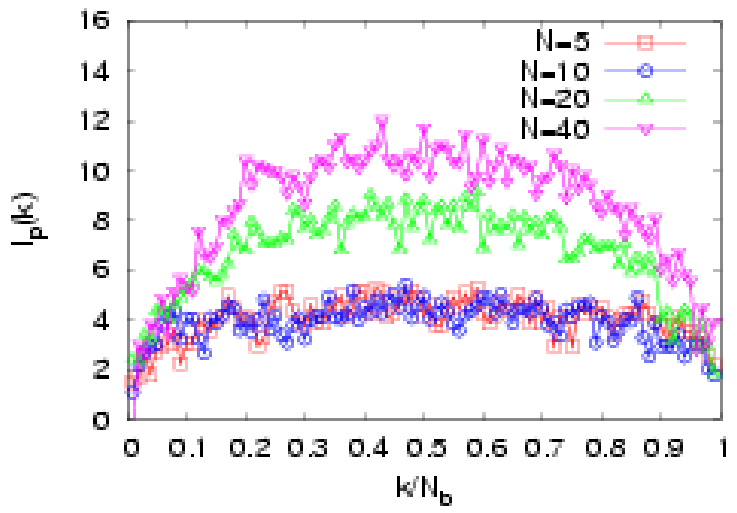

Figure 2. (Colour online) Local persistence length $\mathrm{l}_{\mathrm{p}}(\mathrm{k})$ plotted versus $\mathrm{k} /$ $\mathrm{N}_{\mathrm{b}}$ for $\sigma=0.5, \mathrm{~N}_{\mathrm{b}}=100$, and $\mathrm{T}=3.2$ for different chain lengths $\mathrm{N}$, as indicated

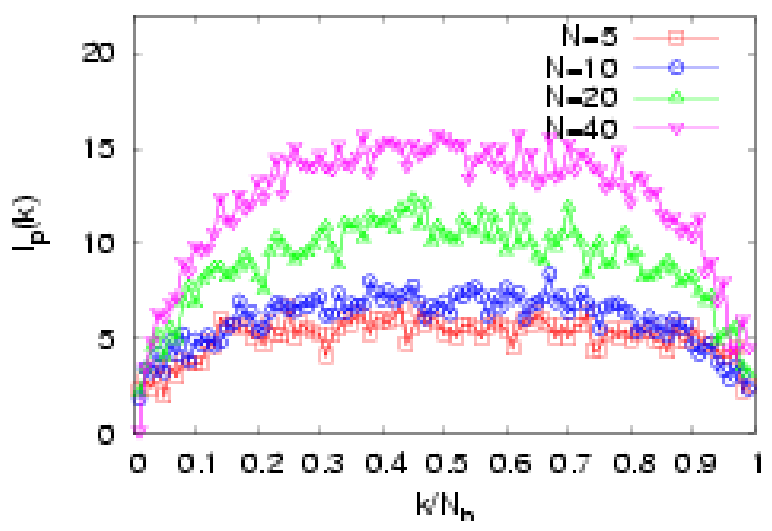

Figure 3. (Colour online) Local persistence length $\mathrm{l}_{\mathrm{p}}(\mathrm{k})$ plotted versus $\mathrm{k} /$ $\mathrm{N}_{\mathrm{b}}$ for $\sigma=0.5, \mathrm{~N}_{\mathrm{b}}=100$, and $\mathrm{T}=4.0$ for different chain lengths $\mathrm{N}$, as indicated

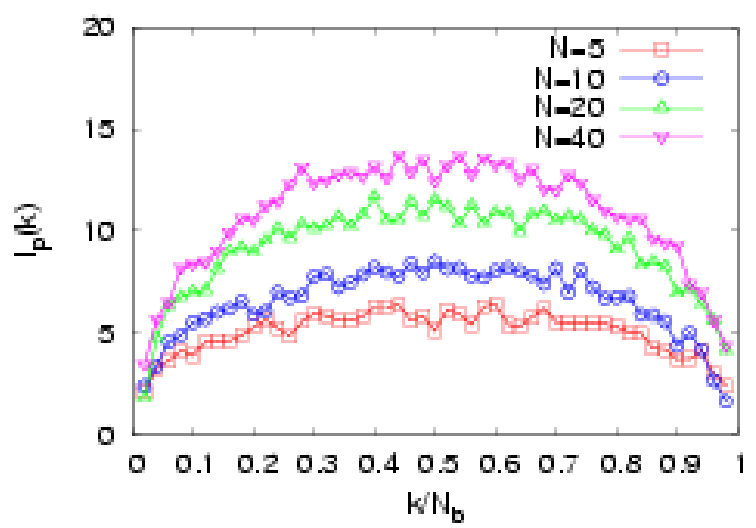

Figure 4. (Colour online) Local persistence length $\mathrm{l}_{\mathrm{p}}(\mathrm{k})$ plotted versus $\mathrm{k} /$ $\mathrm{N}_{\mathrm{b}}$ for $\sigma=1, \mathrm{~N}_{\mathrm{b}}=50$, and $\mathrm{T}=3.2$ for different chain lengths $\mathrm{N}$, as indicated

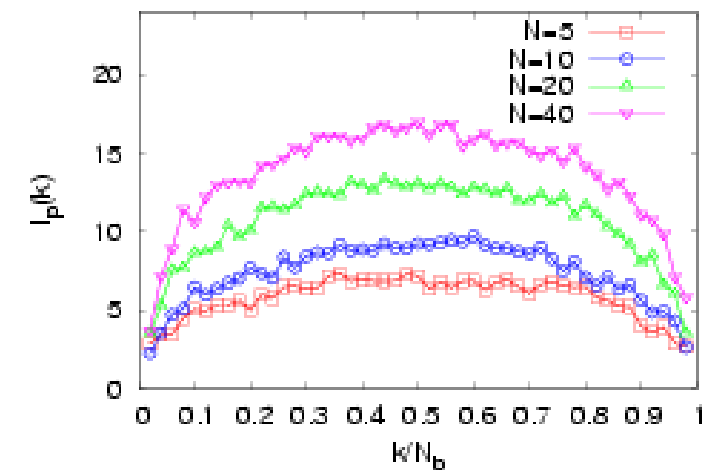

Figure 5. (Colour online) Local persistence length $\mathrm{l}_{\mathrm{p}}(\mathrm{k})$ plotted versus $\mathrm{k} /$ $\mathrm{N}_{\mathrm{b}}$ for $\sigma=1, \mathrm{~N}_{\mathrm{b}}=50$, and $\mathrm{T}=4.0$ for different chain lengths $\mathrm{N}$, as indicated

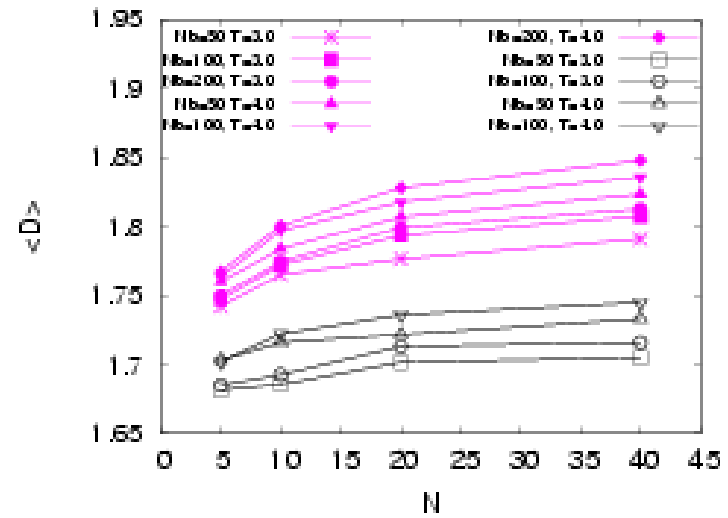

Figure 6. (Colour online) Average dihedral angle along the backbone measured in Radians plotted versus $\mathrm{N}$ for $\sigma=0.5$ (open symbols) and $\sigma=1.0$ (full symbols) for different $\mathrm{N}_{\mathrm{b}}$ and $\mathrm{T}$, as indicated

An estimate of the local stiffness of the backbone could be providedby the average value of dihedral angles formed by four consecutive backbone beads and averaged for all possible such dihedral angles along the backbone, which is shown in Fig. 6.It is seen that increasing the grafting density $\sigma$, curves are considerably shifted to higher values, while the effect of temperature in the range of $\mathrm{T}=3.0$ (close to theta solvent) to $\mathrm{T}=4.0$ (good solvent) is rathers maller. Moreover, for $\mathrm{N}$ in the range $5 \leq N \geq 20$ there is a pronounced increase in $\mathrm{D}$, which is mediatedfor $\mathrm{N}=40$. Data also show a systematic increase in $\mathrm{D}$ as $\mathrm{N}_{\mathrm{b}}$ increases showing that the local stiffness also clearly on average depends on $\mathrm{N}_{\mathrm{b}}$.

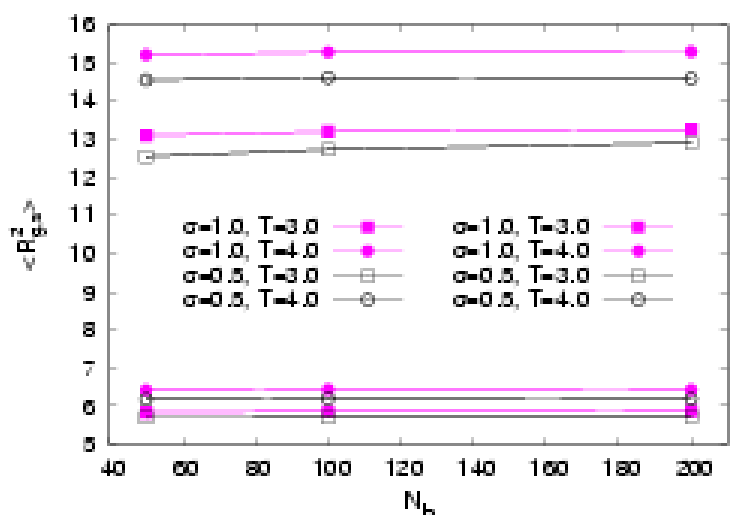

Figure 7. (Colour online) The mean square gyration radius of the side chains versus $\mathrm{N}_{\mathrm{b}}$ for different $\sigma$ and $\mathrm{T}$. Full symbols refer to data with $\mathrm{N}=40$, while open symbols to data with $\mathrm{N}=20$ 
As the persistence length clearly depends on $\mathrm{N}_{\mathrm{b}}$, one could gothe other way around and see if properties of the side chains depend correspondingly on the backbone length $\mathrm{N}_{\mathrm{b}}$. In Fig. 7we plot the mean square gyration radius of the side chains as a function of $\mathrm{N}_{\mathrm{b}}$. While dependencies on $\mathrm{N}, \mathrm{T}$, and obecome apparent and they have been discussed in previous workin terms of effective exponents[15], it is rather difficult to extract a dependenceon $\mathrm{N}_{\mathrm{b}}$. Similar plots we have obtained for other properties ofthe sided chains showing the same behaviour with $\mathrm{N}_{\mathrm{b}}$. It would be interesting to simulate very longbottle brushes, but equilibration problems already impose a limit in the current study, while our result indicate that $\mathrm{N}_{\mathrm{b}}=200$ is alreadyin the regime where such dependence enters a plateau-like regime.

Therefore, a more interesting discussion of the dependence on $\mathrm{N}_{b}$ may focuson the distribution of the monomers, which may indeed reveal some differences between the different cases.Thus,we turn our discussion to the overall shape of bottle-brushpolymers considering properties such as the asphericity andthe acylindricity[43].We follow the description of Theodorou andSuter[53] to define these quantities.Then, the gyration tens or reads as

$$
s=\frac{1}{N_{\xi}} \sum_{i=1}^{N_{\xi}} S_{i} S_{i}^{\mathrm{T}}=\overline{S S^{\mathrm{T}}}=\left[\begin{array}{lll}
\overline{x^{2}} & \overline{x y} & \overline{x z} \\
\overline{x y} & \overline{y^{2}} & \overline{y z} \\
\overline{x z} & \overline{y z} & \overline{z^{2}}
\end{array}\right],
$$

where $\mathrm{S}_{\mathrm{i}}=\operatorname{col}\left(\mathrm{x}_{\mathrm{i}}, \mathrm{y}_{\mathrm{i}}, \mathrm{z}_{\mathrm{i}}\right)$ is the position vector ofeach bead, which is considered with respect to the centre of mass of the beads, and theover-bars denote an average over all beads $\mathrm{N}_{\xi}$. When the gyration tensor of the whole chain is considered, then $N_{\xi}=n N+N_{b}$, where $n$ is the number of the side chain grafted onto the backbone monomers. For the side chains $N_{\xi}$ $=\mathrm{N}$, the gyration tens or and the properties are calculated for each side chain separately and thenan average over all results for each side chain is taken, whilefor the distribution of the backbone beads simply $\mathrm{N}_{\xi}=\mathrm{N}_{\mathrm{b}}$. The gyration tensor is symmetric with real eigenvalues and a Cartesian system that this tensor is diagonal can always be found,

$$
s=\operatorname{diag}\left(\overline{X^{2}}, \overline{Y^{2}}, \overline{Z^{2}}\right),
$$

where the axes are also chosen in such way that the diagonal elements (eigenvalues of s) are in descending order

$$
\overline{X^{2}} \geq \overline{Y^{2}} \geq \overline{Z^{2}} \text {. }
$$

Theseeigenvalues are called the principal moments of the gyration tensor. From the values of the principal moments, one definesquantities such as the asphericity $b$,

$$
b=\overline{X^{2}}-1 / 2\left(\overline{Y^{2}}+\overline{Z^{2}}\right) \text {. }
$$

When the particle distribution is spherically symmetric or has atetrahedral or higher symmetry, then $\mathrm{b}=0$. Theacylindricity c

$$
c=\overline{Y^{2}}-\overline{Z^{2}}
$$

is zero when the particle distribution approaches acylindrical symmetry. Therefore, the acylindricity and asphericityare relevant quantities that would describe some geometricalaspects of the monomer distribution in bottle-brush polymers. These quantities are taken with respect to $s$ that is to the sum of the eigenvalues, i.e., the square gyration radius of the chain,which we also have calculated independently on our original Cartesian coordinate system in order to check our results. Subs cript "s" to quantities $b$ and $c$ are referring to the side chains, "b" to the backbone beads, while $\mathrm{b}$ and $\mathrm{c}$ without subscripts refer to the distribution of allbeads belonging to the bottle-brush mac ro molecule.

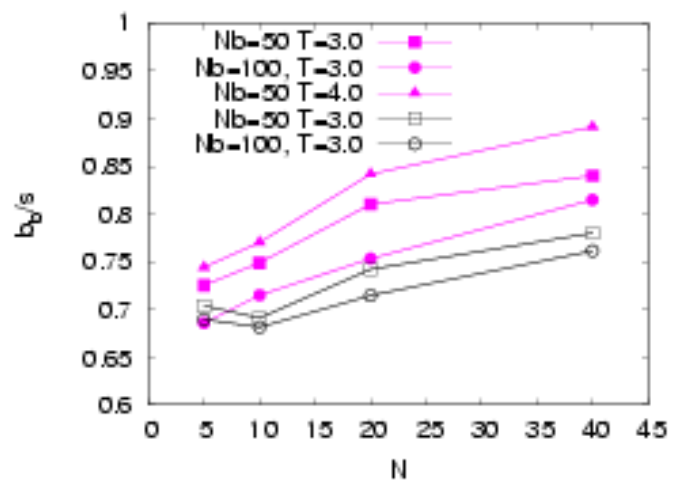

Figure 8. (Colour online) Asphericity of the backbone beads versus $\mathrm{N}$ for $\sigma=1.0$ (full symbols) and $\sigma=0.5$ (open symbols) andvarious cases of $\mathrm{N}_{\mathrm{b}}$ and $\mathrm{T}$ as indicated

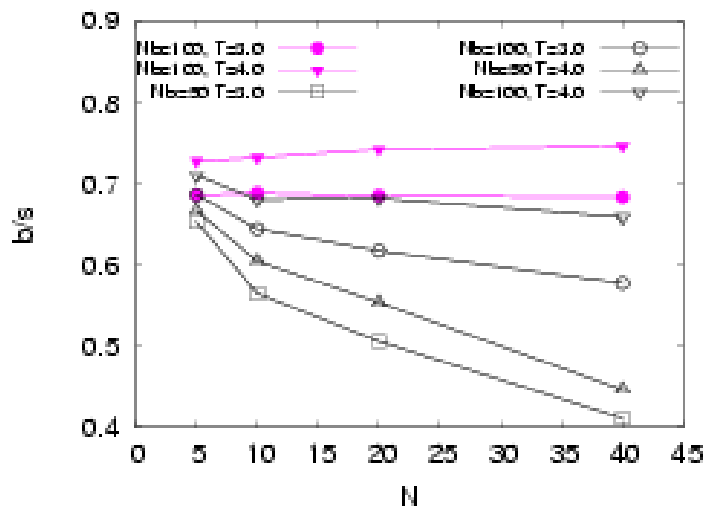

Figure 9. (Colour online) A corresponding plot for the asphericity of the whole bottle-brush molecules is shown

Then, in Fig. 8 the asphericity for the distribution ofthe backbone monomers for various cases is shown. This distribution deviatesconsiderably from a spherical or higher symmetry as expectedfor bottle-brush macromolecules. For $\sigma=1.0$ this effect is more pronounced compared to the case $\sigma=0.5$. Also,increase of the side chain length $\mathrm{N}$ and of the temperature increases the values of $b_{b} / s$. On the other hand, when one takes into account all the monomersfor the calculation of asphericity (Fig. 9), a very s mall variat ion with $\mathrm{N}$ for $\sigma=1.0$ is observed, while for $\sigma=0.5$ thebottlebrush obtains as a whole higher symmetrical structures.This is explained from the fact that the bottlebrush is an elongated object and the increase in the number of side chain monomers favors a spherical or higher symmetry for the whole bottle brush. The effect of temperature is similar.The 
increase of the backbone length $\mathrm{N}_{\mathrm{b}}$ in the results of Figs. 8 and 9 leads to the increase of asphericity for the range of side chain lengths $\mathrm{N}$ we have consideredin our study.

Now if one measures $b_{s} / s$ for each side chain individually and averageover all side chains and plot this data versus $\mathrm{N}_{b}$ gets Fig. 10.Side chains have clearly symmetry close to a spherical one especiallyco mpared to the results of Figs. 8 and 9 and the interestingpoint is that the side chains adopt conformations with higher symmetry as the backbone length $\mathrm{N}_{\mathrm{b}}$ increases. It might suggest that as $\mathrm{N}_{\mathrm{b}}$ exceeds a certain value this dependence should disappear.We should mention here that for $N_{b} \rightarrow \infty$ effects dueto the backbone ends are smeared out. Also, the results for $\mathrm{N}=20$ exhibit higher values of normalized asphericity than those for the case $\mathrm{N}=$ 40 , where the chain is more stretched inthe radial directions from the backbone.

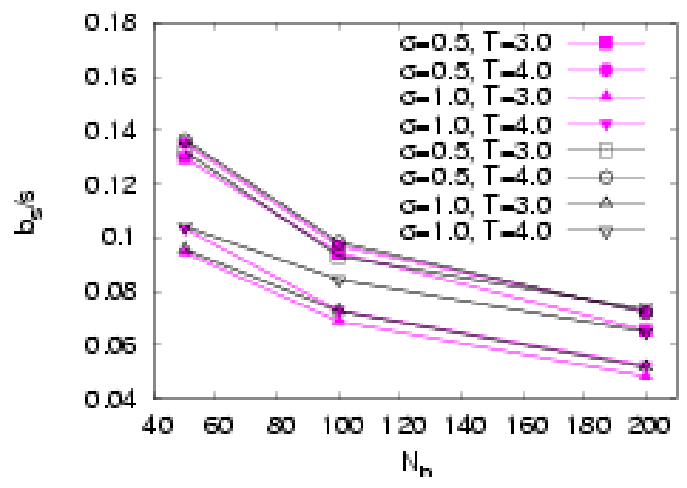

Figure 10. (Colour online) Asphericity of the side chains versus $\mathrm{N}_{\mathrm{b}}$ is plotted for two different side chain lenghts $\mathrm{N}=20$ (full symbols) and $\mathrm{N}=40$ (open symbols) for different $\sigma a n d \mathrm{~T}$ as indicated

In Figs. 11-13 results for the acylindricity of the distribution of the backbone and the side chain monomers is shown. Overall, all distributions shown in Figs. 8-10 validate a cylindrical symmetry for all cases (backbone beads,all beads and the side chain beads). For the distribution ofbackbone monomers (Fig. 11) the increase of temperature $\mathrm{T}$, the backbone length $\mathrm{N}_{\mathrm{b}}$, and the side chain length $\mathrm{N}$ obviously favours a more cylindricalsymmetry of the backbone monomers. The latter is also true for thedistribution of all bottle-brush monomers, but now a more favourable cylindrical symmetry for lower values of $\mathrm{N}_{\mathrm{b}}$ is noted.

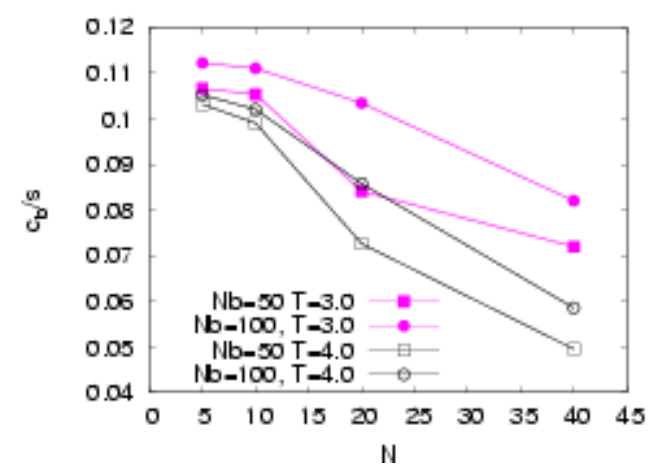

Figure 11. (Colour online) Acylindricity of the backbone beads versus $\mathrm{N}$ for two different temperatures $\mathrm{T}=3.0$ (full symbols) and $\mathrm{T}=4.0$ (open symbols)for $\sigma=1.0$ and different $\mathrm{N}_{\mathrm{b}}$ as indicated

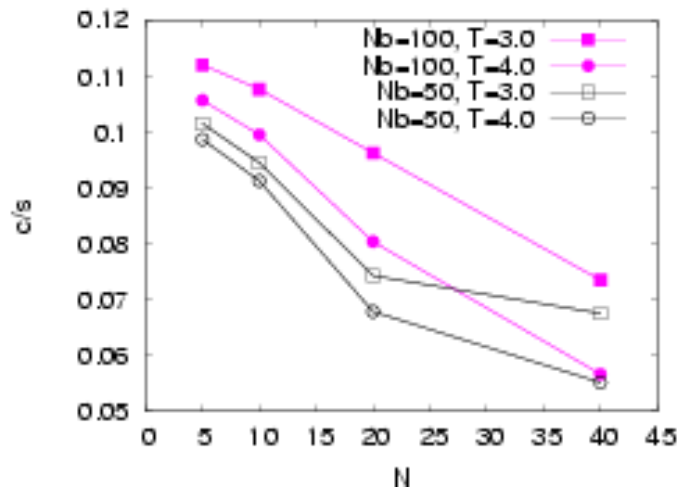

Figure 12. (Colour online) The acylindricity for all the bottle-brush beads is shown for $\sigma=1.0$ and two different $\mathrm{N}_{\mathrm{b}}$, namely $\mathrm{N}_{\mathrm{b}}=100$ (full symbols) and $\mathrm{N}_{\mathrm{b}}=50$ (open symbols) for various temperatures as is shown on theplot

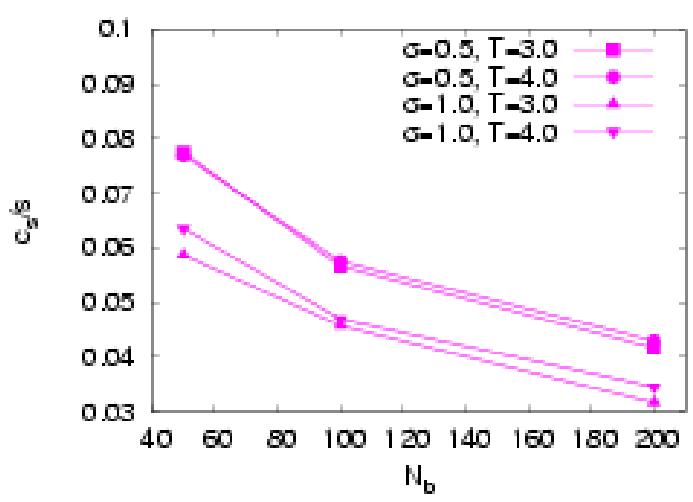

Figure 13. (Colour online) Acylindricity of the side chains versus $\mathrm{N}_{b}$ for the case $\mathrm{N}=20$ and different grafting density oand temperature $\mathrm{T}$, as indicated

For $\mathrm{N}_{\mathrm{b}}=100$ thebottlebrush is able to obtain configurations such as this of Fig. 1c, while for $\mathrm{N}_{\mathrm{b}}=50$ the length of the backbone is rather small for the macromoleculeto allow for such curved structures since the persistence length along the bottlebrushis rather high. The distribution of side chain monomers tends to higher cylindrical symmetryas the grafting density oincreases. In this case also we see that a more cylindrical symmetry is favoured by increasing $\mathrm{N}_{b}$ for the range of values we have studied here. It would be interesting to simulate longer bottle brushes in order to find the limit of $c_{c}$ / $s$ as a function of $\mathrm{N}_{b}$, but this is not possible with the simulation method we have adopted in this study. We note here that although a change of $<\mathrm{R}_{\mathrm{g}, \mathrm{s}}{ }^{2}>$ with $\mathrm{N}_{\mathrm{b}}$ was not seen in Fig. 7, the distributionof the side chain monomers changes as it shown in Figs. 10 and 13 obtaininga more cylindrical shape with the increase of the backbone length. One should, however, also keep in mind that the number of side chains that are in the middle of the backbone is higher as thebackbone length increases, and these side chains are more stretched in the radial directions from the backbone, suggesting that the higher cylindrical symmetry with increased $\mathrm{N}_{\mathrm{b}}$ can be also attributed to this reason.

\section{Conclusions}


In summary, we have demonstrated that the interplay of solvent quality, grafting density, side chain and backbone length in bottle brushes gives rise to very rich structural properties, where the distribution of monomers exhibit differences between bottle brushes, whereas other quantities would not reveal any dependence. A coarse-grained bead-spring model for bottle-brush polymers was studied via mo lecular dynamics, by vary ing both the chain length $\mathrm{N}_{\mathrm{b}}$ of the backbone and the side chains $\mathrm{N}$, for two values of the grafting density, under variable solvent conditions. The main target of the present work was to emphasize this geometric description of the shape of bottle-brush macromolecules at temperatures close to theta solvent conditions and in the good solvent regime. A short discussion on a way to extract the persistence length in bottle brushes and its relevant drawbacks was also briefly discussed for the present model.

\section{ACKNOWLEDGEMENTS}

P.E.T. would like to thank Profs. K. Binder and W. Paul for an exceptional collaboration over the last years. This work has benefitted by their insight and discussions. He also acknowledges financial support by the Austrian Science Foundation within the SFB ViCoM (Grant No. F41).N.G.F. has been partly supported by MICINN, Spain, through Research Contract No. FIS2009-12648-C03.

\section{REFERENCES}

[1] M.Zhang, A. H.E. Muller, Polym. Sci. Part. A: Polym. Chem., 43, 3461 (2005).

[2] S.S. Sheiko, B.S. Sumerlin, K. Matyjaszewski, Progr. Polym. Sci. 33, 759 (2008).

[3] H.-P. Hsu, W. Paul, K. Binder, Macromol. Theory Simul. 16, 660 (2007).

[4] H.-P. Hsu, W. Paul, K. Binder, Macromol. Theory Simul. 20, $510(2011)$.

[5] I.I. Potemkin, V.V. Palyulin, Polym. Sci., Ser. A, 51, 123 (2009).

[6] A.V. Subbotin, A. N. Semenov, Polym. Sci., Ser. A, 49, 1328 (2007).

[7] C. Li, N. Gunari, K. Fischer, A. Janshoff, M. Schmidt, Angew. Chem. Int. Ed., 43, 1101 (2004).

[8] Proteoglycans: Structure, Biology, and Molecular Interactions, edited by R. V. Iozzo (Marcel Dekker, New York, 2000).

[9] J. Klein, Science, 323, 47 (2009).

[10] P.E. Theodorakis, W. Paul, K. Binder, Europhys. Lett., 88, 63002 (2009).

[11] P.E. Theodorakis, W. Paul, K. Binder, J. Chem. Phys., 133,
104901 (2010).

[12] P.E. Theodorakis, W. Paul, K. Binder, Macromolecules, 46, 5137 (2010).

[13] I. Erukhimovich, P.E. Theodorakis, W. Paul, K. Binder, J. Chem. Phys., 134, 054906 (2011).

[14] P.E. Theodorakis, W. Paul, K. Binder, Eur. Phys. J. E, 34, 52 (2011).

[15] P.E. Theodorakis, H.-P. Hsu, W. Paul, K. Binder, J. Chem. Phys., 135, 164903 (2011).

[16] P. J. Flory, Statistical Mechanics of Chain Molecules (Interscience, New York, 1969).

[17] A. Yu. Grosberg, A. R. Khokhlov, Statistical Physics of Macromolecules (American Institute of Physics, New York, 1994).

[18] M. Rubinstein, R. H. Colby, Polymer Physics (Oxford University Press, Oxford, 2003).

[19] H.-P. Hsu, W. Paul, K. Binder, Macromolecules, 43, 3094 (2010).

[20] H.-P. Hsu, W. Paul, K. Binder, Europhys. Lett., 92, 2803 (2010).

[21] K. Fischer, M. Gerle, M. Schmidt, Proc. ACS, PMSE Anaheim, 30, 133 (1999).

[22] K. Fischer, M. schmidt, Macromol. Rapid Commun., 22, 787 (2001).

[23] B. Zhang, F. Grohn, J.S. Pedersen, K. Fischer, M. Schmidt, Macromolecules, 39, 8440 (2006).

[24] G. Cheng, Y.B. Melnichenko, G.D. Wignall, F. Hua, K. Hong, J.W. Mays, Macromolecules, 41, 9831 (2008).

[25] T. M. Birshtein, O.V. Borisov, E.B. Zhulina, A.R. Khokhlov, T.A. Yurasova, Polym. Sci. U.S.S.R., 29, 1293 (1987).

[26] Y. Rouault, O. V. Borisov, Macromolecules, 29, 2605 (1996).

[27] M. Saariaho, O. Ikkala, I. Szleifer, I. Erukhimovich, G. ten Brinke, J. Chem. Phys., 107, 3267 (1997).

[28] K. Shiokawa, K. Itoh, N. Nemoto, J. Chem. Phys., 111, 8165 (1999).

[29] P.G. Khalatur, D.G. Shirvanyanz, N.Yu. Starovoitova, A.R. Khokhlov, Macromol. Theory Simul., 9, 141 (2000).

[30] S. Elli, F. Ganazzoli, E.G. Timoshenko, Y.A. Kuznetsov, R. Connolly, J. Chem. Phys., 120, 6257 (2004).

[31] A. Yethiraj, J. Chem. Phys., 125, 204901 (2006).

[32] H.-P. Hsu, W. Paul, S. Rathgeber, K. Binder, Macromolecules, 43, 1592 (2010).

[33] L. Feuz, F.A.M. Leermakers, M. Textor, O.V. Borisov, Macrmolecules, 38, 8901 (2005).

[34] G.H. Fredrickson, Macromolecules, 26, 2825 (1993).

[35] S.S. Sheiko, O.V. Borisov, S.A. Prokhorova, M. Moller, Eur. Phys. J. E, 13, 125 (2004).

[36] V.V. Vasilevskaya, A.A. Klochkov, P.G. Khalatur, A.R. Khokhlov, G. ten Brinke, Macromol. Theory Simul., 10, 389 
(2001).

[37] G.S. Grest, K. Kremer, Phys. Rev. A, 33, 3628 (1986).

[38] M. Murat, G.S. Grest, Macromolecules, 22, 4054 (1989).

[39] P.E. Theodorakis, N.G. Fytas, Soft Matter, 7, 1038 (2011).

[40] G.S. Grest, M. Murat, in Monte Carlo and Molecular Dynamics Simulations in Polymer Science, ed. by K. Binder (Oxford Univ. Press, New York, 1995), p. 476.

[41] P.E. Theodorakis, N.G. Fytas, Europhys. Lett., 93, 43001 (2011).

[42] N.G. Fytas, P.E. Theodorakis, J. Phy s.: Condens. Matter, 23, 505104 (2011).

[43] P.E. Theodorakis, N.G. Fytas, J. Chem. Phys., 136, 094902 (2012).

[44] D.N. Theodorou, in Simulation Methods for Polymers, ed. by M.Kotely anskii (Monticello, New York, 2004).

[45] Computationa Soft Matter: From Synthetic Polymers to Proteins\}, ed. by N. Attig, K. Binder, H. Grubmuller, K.
Kremer (John von Neumann-Institute for Computing (NIC), Julich, Germany, 2004).

[46] G.S. Grest, M. Murat, Macromolecules, 26, 3108 (1993).

[47] W.W. Graessley, R.C. Hayward, G.S. Grest, Macromolecules, 32, 3510 (1999).

[48] T. Kreer, S. Metzger, M. Muller, K. Binder, J. Baschnagel, J. Chem. Phys., 20, 4012 (2004).

[49] K. Binder, T. Kreer, A. Milchev, Soft Matter, 7, 7159 (2011).

[50] W.F. van Gunsteren, H.J.C. Berendsen, Mol. Simul., 1, 173 (1988).

[51] Information about algorithms and implementation details for the Gromacs package can found at http://www.gromacs.org; H.J.C. Berendsen, D. van der Speol, R. van Drunen, Comp. Phys. Comm. 91, 43 (1995); E. Lindahl, B. Hess, D. van der Spoel, J. Mol. Mod. 7, 306 (2001).

[52] L. Schafer, K. Elsner, Eur. Phys. J. E, 13, 225 (2004).

[53] D.N. Theodorou, U.W. Suter, Macromolecules, 18, 1206 (1985). 